\title{
Type-1 echoes from the mid-latitude $E$-Region ionosphere
}

\author{
C. Haldoupis ${ }^{1}$, D. T. Farley ${ }^{2}$, K. Schlegel ${ }^{3}$ \\ ${ }^{1}$ Physics Department, University of Crete, Iraklion, Greece \\ ${ }^{2}$ School of Electrical Engineering, Cornell University, Ithaca, NY, USA \\ ${ }^{3}$ Max-Planck-Institut für Aeronomie, Katlenburg-Lindau, Germany
}

Received: 3 June 1996 / Revised: 2 October 1996 / Accepted: 4 October 1996

\begin{abstract}
This paper presents more data on the properties of type-1 irregularities in the nighttime midlatitude $\boldsymbol{E}$-region ionosphere. The measurements were made with a $50-\mathrm{MHz}$ Doppler radar system operating in Crete, Greece. The type-1 echoes last from several seconds to a few minutes and are characterized by narrow Doppler spectra with peaks corresponding to wave phase velocities of $250-350 \mathrm{~m} / \mathrm{s}$. The average velocity of $285 \mathrm{~m} / \mathrm{s}$ is about $20 \%$ lower than nominal $\boldsymbol{E}$ region ion-acoustic speeds, probably because of the presence of heavy metallic ions in the sporadic- $\boldsymbol{E}$-layers that appear to be associated with the mid-latitude plasma instabilities. Sometimes the type- 1 echoes are combined with a broad spectrum of type- 2 echoes; at other times they dominate the spectrum or may appear in the absence of any type- 2 spectral component. We believe these echoes are due to the modified two-stream plasma instability driven by a polarization electric field that must be larger than $10 \mathrm{mV} / \mathrm{m}$. This field is similar in nature to the equatorial electrojet polarization field and can arise when patchy nighttime sporadic- $\boldsymbol{E}$-layers have the right geometry.
\end{abstract}

\section{Introduction}

Schlegel and Haldoupis (1994) reported the first evidence of type-1 irregularities in the mid-latitude $\boldsymbol{E}$ region ionosphere. These are meter-scale electrostatic plasma waves generated directly by the modified twostream, or Farley-Buneman (F-B), instability (Farley, 1963; Buneman, 1963) when relative electron-ion drift speeds near the altitude of $105 \mathrm{~km}$ exceed the plasma ion-acoustic speed $\boldsymbol{C}_{s}$. The backscatter from these waves is characterized by a narrow spectral peak having a Doppler velocity that equals approximately $\boldsymbol{C}_{s}$. This spectral signature is distinctly different from the broad type-2 spectrum, which has a much smaller mean Doppler and is associated with a turbulent cascade from long wavelengths to short.
Type-1 waves occur regularly in equatorial and auroral latitudes where strong horizontal electrojets exist because the ambient electric fields there attain relatively large values (see reviews by Fejer and Kelley, 1980, and Haldoupis, 1989). On the other hand, type-1 backscatter is not expected at mid-latitudes where typical electric fields are only a few $\mathrm{mV} / \mathrm{m}$, well below the threshold required for excitation of the modified two-stream instability (e.g., Kelley, 1989). The observation of Schlegel and Haldoupis (1994), however, implied the existence at times, of mid-latitude electric fields at least as large as $15 \mathrm{mV} / \mathrm{m}$, i.e., at least an order of magnitude larger than expected.

This first mid-latitude type-1 observation was made by SESCAT in its early days of operation. SESCAT, an acronym for sporadic- $\boldsymbol{E}$ Scatter experiment, is a 50 $\mathrm{MHz}$ continuous-wave radio Doppler system that has been operating in Crete, Greece since 1992. It monitors continuously the backscatted from a fixed $\boldsymbol{E}$-region volume centered at about $30.8^{\circ}$ geomagnetic latitude. After long periods of SESCAT operation and numerous echo recordings, we are now in a position to present more evidence on mid-latitude type-1 echoes and their statistics. We also discuss further the origin of the large electric fields that we believe (Haldoupis et al., 1996) are responsible for mid-latitude type-1 wave generation.

\section{The experiment}

The experimental system is a continuous-wave, $(\mathrm{CW})$, 50.52-MHz bistatic Doppler radar capable of observing coherent backscatter from 3-m, magnetic aspect sensitive plasma irregularities in the mid-latitude $\boldsymbol{E}$-region ionosphere. The transmitter and receiver are separated by about $120 \mathrm{~km}$ along the northern coast of Crete $(\sim$ $35^{\circ}$ geographic latitude, $24^{\circ}$ to $25^{\circ}$ geographic longitude and $\sim 28^{\circ}$ geomagnetic latitude) and have their antenna arrays, each of $8^{\circ}$ beamwidth, beaming northward and intersecting to form a fixed target region in the $\boldsymbol{E}$-layer where the propagation vector $\boldsymbol{k}_{\text {incident }}-\boldsymbol{k}_{\text {scattered }}$ is 
perpendicular to the earth's magnetic field. The 3-dB viewing region of about $20 \mathrm{~km}$ in longitude and $40 \mathrm{~km}$ in latitude is located over the southern Aegean sea with its center at about $30.8^{\circ}$ invariant magnetic latitude $(\boldsymbol{L}=1.35), 52.5^{\circ}$ magnetic dip, and $2.5^{\circ}$ magnetic declination. The transmitted power can be set in the range from 400 to $800 \mathrm{~W}$.

In the SESCAT configuration the mean observing direction is about $8^{\circ}$ east of geographic north, or $\sim 5.5^{\circ}$ east of geomagnetic north; that is, SESCAT observes nearly along the north-south direction and therefore detects only meridional motions. For the altitudes of interest between 100 and $115 \mathrm{~km}$, these motions are controlled primarily by zonal electric fields, because plasma motions are mainly due to $\boldsymbol{E} \times \boldsymbol{B}$ electron drifts. North-south neutral-wind components may also have some effect, but they are not important for our discussion here. Further details on SESCAT are given by Haldoupis and Schlegel (1993).

In the first year of operation, the experiment was run on a campaign basis for time-periods of a few days because of limitations imposed by slow data acquisition and analysis procedures. In one of these data-collection periods the first observation of mid-latitude type-1 echoes was made (Schlegel and Haldoupis, 1994). Later, a PC-based digital signal processor (DSP) unit was employed and software was developed that allowed fast Fourier transformations and power-spectrum calculations to be performed with high efficiency in real time. In this mode, a digitization rate of $2.5 \mathrm{kHz}$ was adopted that led to a sampling interval of $0.4 \mathrm{~ms}$ and a Nyquist frequency of $1.25 \mathrm{kHz}$. For the SESCAT system, zero Doppler shift corresponds to a frequency offset of exactly $1 \mathrm{kHz}$ because the transmitter and receiver local oscillator frequencies differ by $1 \mathrm{kHz}$, a technique developed by Koechler et al., (1985) that allows measurement of both positive and negative Doppler displacements. Single power spectra are obtained by Fourier transforming a 2048-point time-series of $0.8192 \mathrm{~s}$, which yields a frequency resolution of 1.23 $\mathrm{Hz}$, or a phase-velocity resolution of $3.85 \mathrm{~m} / \mathrm{s}(1 \mathrm{~Hz}=$ $3.12 \mathrm{~m} / \mathrm{s})$. The output spectra, stored on a disk, are averages of six single spectra, and so sequential Doppler spectra are available for further analysis every $4.9152 \mathrm{~s}$

\section{Observed characteristics of mid-latitude type-1 echoes}

A recent statistical study by Haldoupis and Schlegel (1996) showed that SESCAT echoes occur primarily in the summer nighttime in close connection with intense sporadic $\boldsymbol{E}_{s}$ layers. The backscatter is usually characterized by rather small Doppler velocities of less than 50 to $100 \mathrm{~m} / \mathrm{s}$, which suggests that the irregularities are secondaries generated indirectly, probably by a nonlinear cascade from larger scale gradient drift plasma waves. This statistical study, however, showed also that there is a tail in the mean Doppler velocity distribution that extends to velocities higher than $200 \mathrm{~m} / \mathrm{s}$.

A spectrogram analysis of several hundred hours of SESCAT backscatter, mostly from 1993-1995 summer nighttime intervals, revealed that mid-latitude type-1 echoes constitute a small but distinct fraction of the observations. These echoes are identified by the presence of a narrow peak in the spectrum at a Doppler velocity well above $200 \mathrm{~m} / \mathrm{s}$. They appear sporadically during active periods of regular type- 2 echo occurrence most commonly during quasi-periodic sequences of strong backscatter bursts in the dark hours prior to local midnight. Usually the type-1 echoes are fairly weak and are imbedded in a broad, low-velocity type- 2 spectrum with negative Doppler shifts (northward motion). Less common is the case of a dominant type-1 echo with no, or little, type- 2 spectral component present, that is, they occur without, or little, relation to type- 2 echoes.

These basic features are illustrated in Fig. 1, which is a SESCAT spectrogram taken from a very active night of mid-latitude scatter (local time is UT $+1.6 \mathrm{~h}$ ). The signal intensity changes quasi-periodically with a typical period around $10 \mathrm{~min}$ and in a fashion similar to the quasi-periodic echoes observed regularly by the middle and upper (MU) atmosphere radar in Kyoto, which is located at about the same geomagnetic latitude as SESCAT and operates at the same frequency (e.g., Yamamoto et al., 1991). As seen from the mean and spectral peak-velocity time-sequences in the upper panel of Fig. 1, the echo intensity variations are followed rather closely, although not always in phase, by a wavelike variation in Doppler velocity. Actually, the velocity fluctuates mostly between $\pm 100 \mathrm{~m} / \mathrm{s}$, in good agreement with similar $50-\mathrm{MHz}$ observations reported before by Ecklund $\boldsymbol{e t}$ al. (1981) and Riggin $\boldsymbol{e t}$ al. (1986).

Figure 1 shows that the observed spectra are basically type 2, that is, rather broad with their mean (or peak) Doppler velocities near and around zero Doppler shift. It is also evident from the spectrogram, however, that often the spectrum broadens and becomes skewed with some power leaking out to velocities exceeding $\pm 250 \mathrm{~m} / \mathrm{s}$. Inspection of sequential spectra during these times shows that weak type-1 peaks do appear occasionally for short times inside the much stronger type-2 spectrum. Also seen in Fig. 1 are a few cases of dominant narrow spectral components at large negative Doppler velocities occurring at times of reduced type-2 echo activity.

For example, a well-defined and dominant type-1 echo is observed at about 2055 (20.9 h) UT. These spectra are shown in more detail in Fig. 2. From 2054 to 2056 UT, the scatter has a very narrow spectrum centered at about $-360 \mathrm{~m} / \mathrm{s}$ (wave motions away from the radar). This echo appears to be unrelated to the lowvelocity type-2 scatter that is present also in the spectrogram (it is important to stress here that the reduced type- 2 echo activity is not a result of normalization). Shown in Fig. 2 is also a narrow but very faint belt of backscatter seen at about $+430 \mathrm{~m} / \mathrm{s}$ which occurs at the same time as the strong type- 1 echoes seen at negative Doppler shifts. This is a true observation, representing a signal signature and not an artifact of the system, which indicates the presence inside the radar field of view also of very weak but fast-moving type-1 waves in directions toward the radar (positive shifts). At 


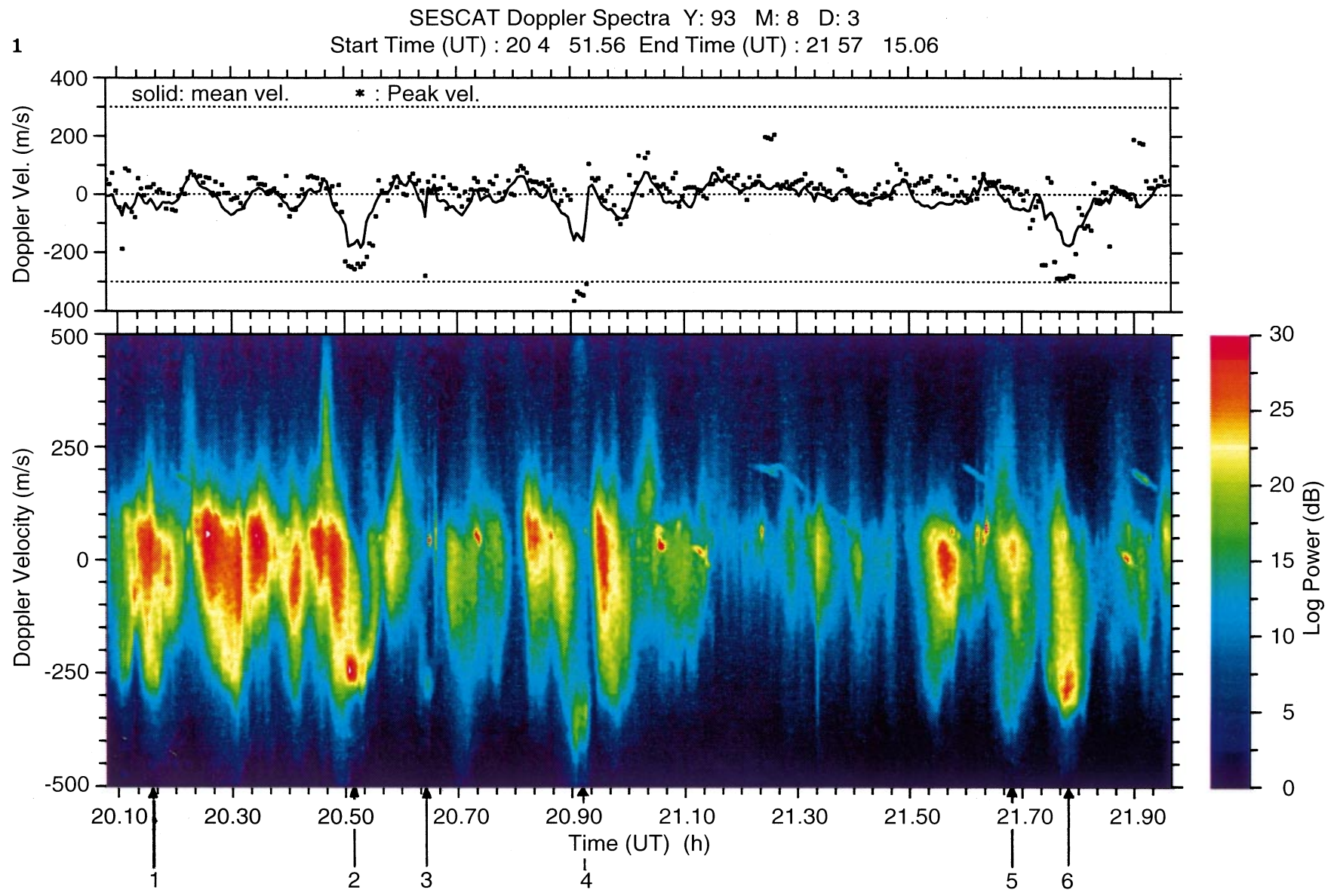

Fig. 1. A spectrogram from an active period of nighttime mid-latitude $50-\mathrm{MHz}$ backscatter. The signal bursts are associated primarily with broad spectra of type-2 like echoes. At the times shown with vertical bars and numbered along the time-axis, however, we also saw type-1 echoes, characterized by narrow spectral peaks at velocities higher than $250 \mathrm{~m} / \mathrm{s}$ (see Fig. 3). Note that the times are in decimal hours, not hours and minutes, but the tick marks are every 2 min

along the observing direction. Figure 4 shows that the abrupt jump in peak velocity that marks the onset of dominant type-1 echoes is consistent with the notion of a threshold required for the excitation of F-B waves. Furthermore, the relative invariability of type-1 velocities near $-350 \mathrm{~m} / \mathrm{s}$, as seen from the peak-velocity changes in the upper panel, is in good agreement with the well-known observational property of equatorial and auroral type-1 waves, namely that their phase velocity remains fairly constant near the plasma ionacoustic speed.

In addition to the dominant type-1 cases in Fig. 4, there are also some weak type-1 echoes present between 2107 and 2109 UT which are hidden inside the simultaneous intense backscatter burst of low velocities (type 2). These weak type-1 echoes and their evolution during an intensifying backscatter burst are identified better in time-stacks of sequential self-normalized spectra. An example taken from a different event is shown in Fig. 5, where time increases downward and each spectrum is averaged over $14.75 \mathrm{~s}$. There is clear excitation of type-1 waves near spectra 10 and 25 .

Our data suggest that weak type- 1 echoes originate in limited regions where the electric field has intensified 


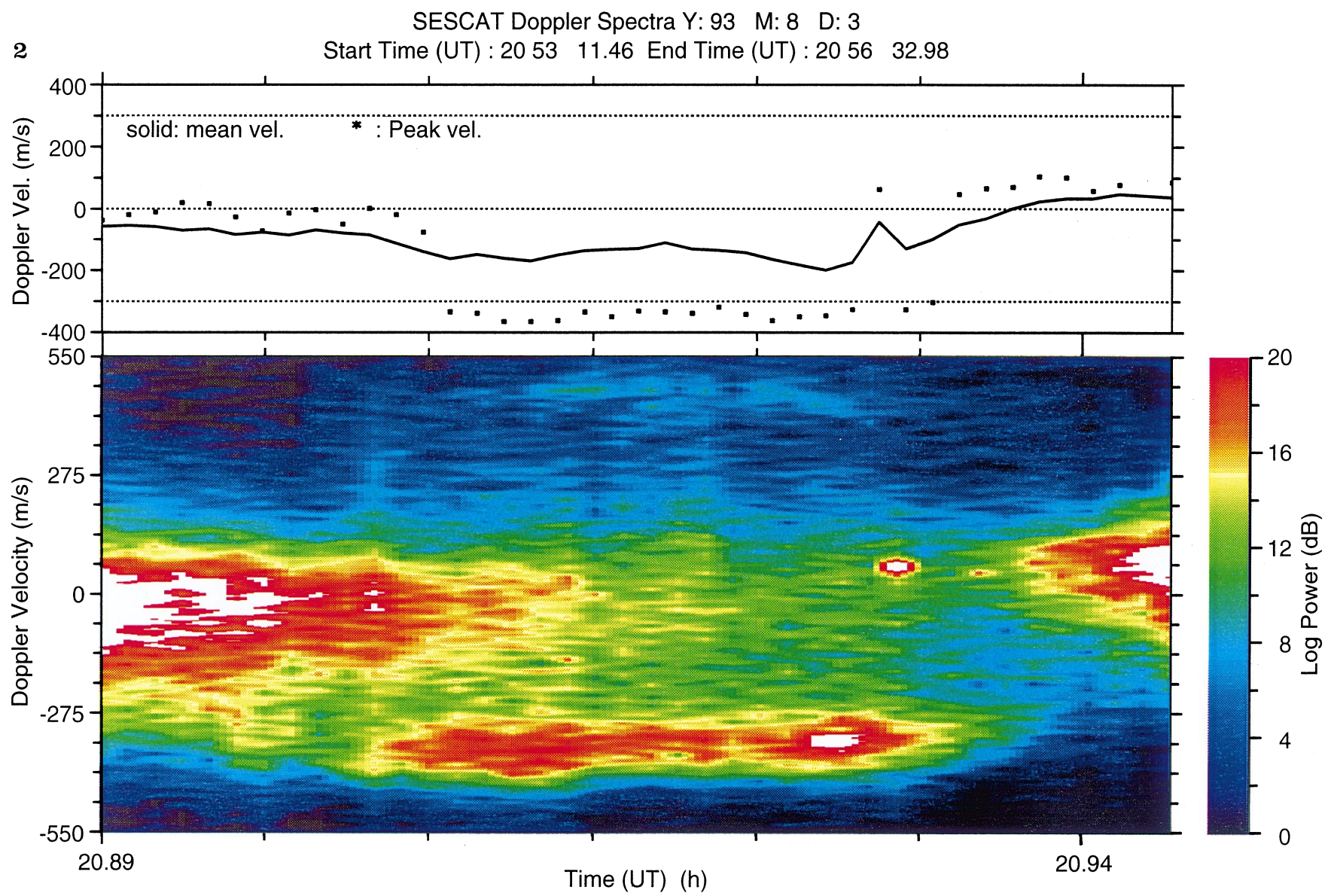

Fig. 2. An expanded portion of Fig. 1 (30-s tick marks), centered near 2055 UT, that shows a pronounced type-1 event appearing abruptly and lasting for about $2 \mathrm{~min}$. This scatter, which seems to be independent of the simultaneous type- 2 echoes present, exhibits a narrow spectrum with a peak velocity of about $-360 \mathrm{~m} / \mathrm{s}$ (northward). A very weak belt of scatter is also seen concurrently at positively shifted velocities somewhat above $+400 \mathrm{~m} / \mathrm{s}$

weighted mean is $2.5 \mathrm{~min}$. The longer-lived events (the longest recorded was $8.5 \mathrm{~min}$ ) are associated with dominant type-1 echoes. The weak type-1 echoes usually last for about a minute or less and often appear periodically, in close association with the quasi-periodic type-2 scatter that occurs regularly in the summer premidnight hours. According to one hypothesis, the quasi-periodic echoes relate to altitude modulated $\boldsymbol{E}_{s^{-}}$ layers caused by the passage of a quasi-monochromatic gravity wave (e.g., Woodmann et al., 1991; Tsunoda et al., 1994).

\section{The type-1 phase velocities}

related with intense and strongly structured sporadic- $\boldsymbol{E}$ layers (e.g., Riggin $\boldsymbol{e t}$ al., 1986, and references therein), we suggest that the electric field intensifications implied here are of local origin.

Figure 6 presents a histogram showing the distribution of the durations of 35 type- 1 events encountered in the SESCAT recordings during the 1993 and 1994 summer months. The statistics here include both "weak" and "dominant" type-1 echoes, as already defined. The great majority of event lifetimes are rather short, less than a couple of minutes, whereas the overall
The overwhelming majority of type-1 echoes occurred in the nighttime sector prior to local midnight, always with negative Doppler shifts; that is, the scattering waves propagated northward and upward perpendicular to $\boldsymbol{B}$. So far we have seen only one pronounced event of dominant, positively shifted type-1 waves. Interestingly, this event occurred in the postmidnight time-sector, when SESCAT also observes a reversal in mean velocity (Haldoupis and Schlegel, 1996). The observed velocity 


\section{SESCAT Doppler Spectra \\ $Y: 93 \quad M: 8 \quad D: 3 \quad$ Integration time (s) : 9.830}

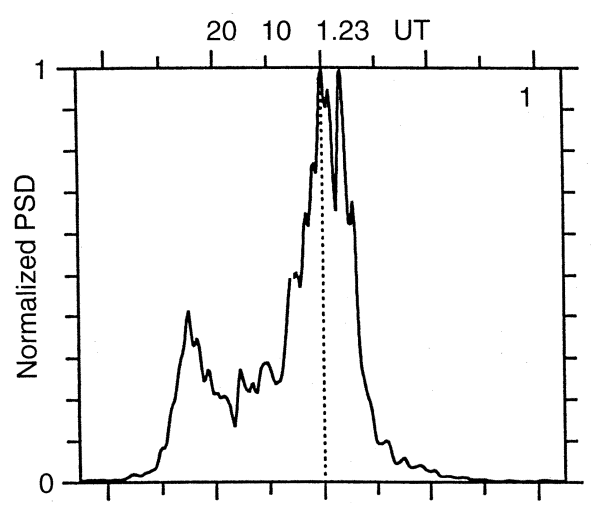

$\begin{array}{llll}20 & 32 & 3.39 & \text { UT }\end{array}$
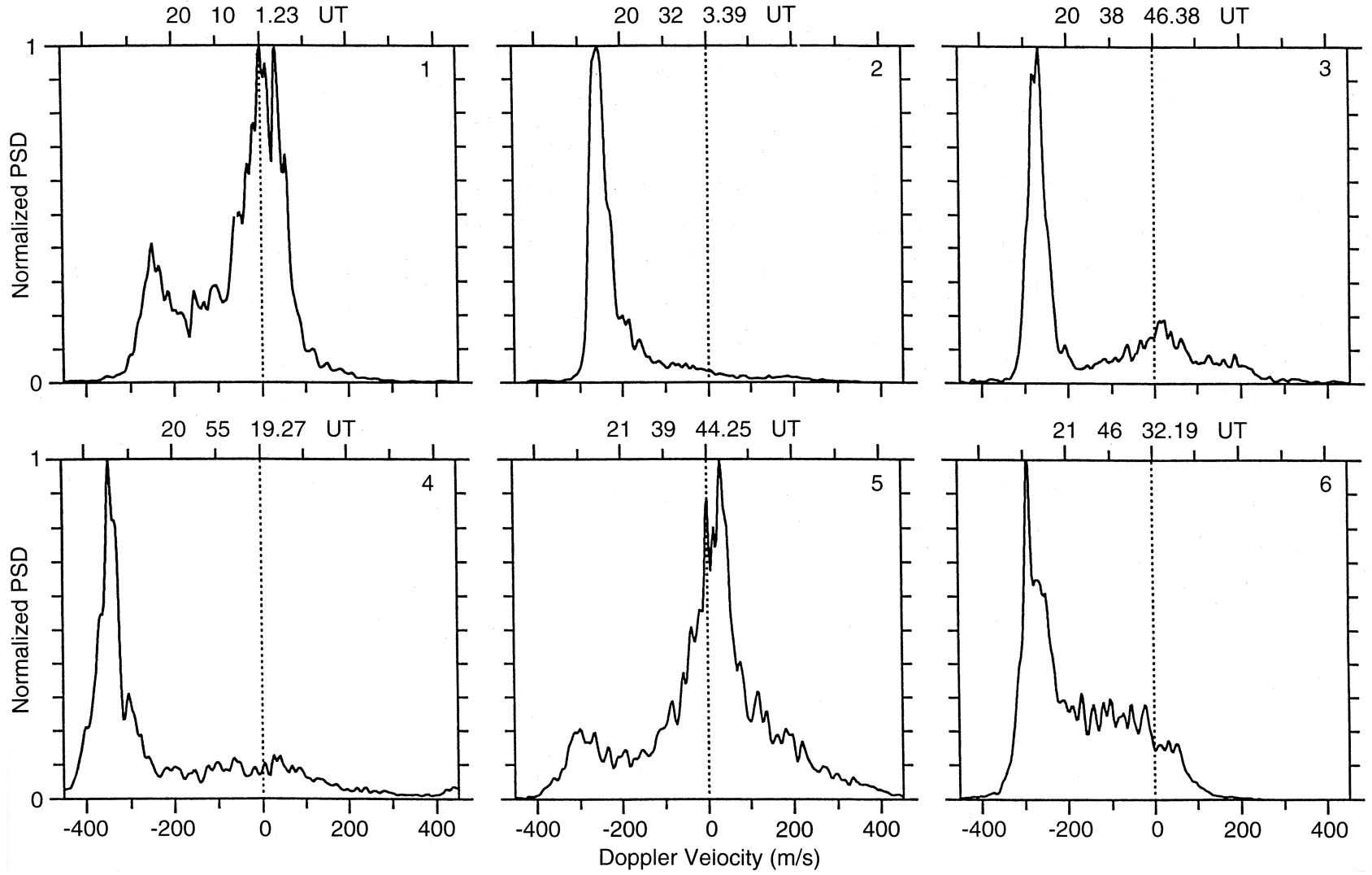

Fig. 3. Six examples of self-normalized power spectra corresponding to the times marked on Fig. 1. We see the characteristic Doppler spectrum signature identified with Farley-Buneman waves in the

polarity and time of occurrence is presumably related closely to the nature of large electric fields responsible for the excitation of the F-B instability. We will return to this point later; here, we focus on the magnitude of the measured velocities.

The statistics to be shown are based on several type-1 events which are accompanied by little if any type- 2 echoes. Figure 7 shows in the upper panel the distribution of peak-velocity magnitude. About $96 \%$ of the histogram points are due to negatively Doppler-shifted signals. The lower panel shows the distribution of spectral width (estimated from the second spectral moment) to peak-velocity ratio. As seen, this ratio is small (the mean value is 0.27 ), in line with equatorial type-1 scatter (e.g., Fejer and Kelley, 1980; Sudan, 1983)

In Fig .7 we use the peak velocity instead of mean velocity because it is a less biased and thus a more representative measure of the type- 1 wave phase velocity. One should be aware, of course, that in the radar frame of reference the frequency shifts can be affected by neutral winds, but this effect should be minor for the type-1 echoes. For example, the TIME-GCM (timegeneral circulation model) neutral-wind model of Roble and Ridley (1994), which is currently regarded as the most advanced and realistic model, suggests for the SESCAT viewing location an average neutral-wind
$E$-region plasma. These echoes are mixed with a broad spectrum at lower velocities, probably because of spatial and/or temporal averaging

contribution of about $\pm 20 \mathrm{~m} / \mathrm{s}$ along the radar observing direction (for details see Haldoupis and Schlegel, 1996).

Figure 7 shows that the observed type- 1 velocities range from 230 to $370 \mathrm{~m} / \mathrm{s}$, but most are below $300 \mathrm{~m} / \mathrm{s}$ and the mean is about $285 \mathrm{~m} / \mathrm{s}$. This mean is roughly $20 \%$ lower than the (molecular) ion-acoustic speed near $105 \mathrm{~km}$, where plasma conditions are optimum for twostream excitation, and is also substantially lower than the equatorial type-1 velocities, which average at about $350 \mathrm{~m} / \mathrm{s}$ (e.g., Fejer and Kelley, 1980). The lower velocity at mid-latitudes is probably due primarily to the presence of heavy metallic ions in the nighttime sporadic- $\boldsymbol{E}$-layers.

Fluid theory predicts that type- 1 waves are generated when the electron-ion drift velocity exceeds $\mathrm{C}_{\mathrm{s}}(1+\psi)$, where $\mathrm{C}_{\mathrm{s}}$ is the ion-acoustic speed $\sqrt{K_{B}\left(T_{e}+T_{i}\right) / \bar{m}_{i}}, \boldsymbol{K}_{B}$ is Boltzmann's constant, $\bar{m}_{i}$ is the mean ion mass, $\psi=$ $\boldsymbol{v}_{e} v_{i} \Omega_{e} \Omega_{i}$, with the subscripted $\boldsymbol{T}, \boldsymbol{v}$, and $\Omega$ being the temperatures, collision frequencies with neutrals, and gyrofrequencies for the electrons and the ions. At threshold, the type-1 phase velocity is just $\boldsymbol{C}_{s}$, and we assume, as at the equator (e.g., Farley, 1985) and probably in the auroral zone, that the actual measured velocities are always close to this threshold value.

As pointed out first by Schlegel (1985), a significant reduction in $\boldsymbol{C}_{S}$ can result from an increase in the mean 


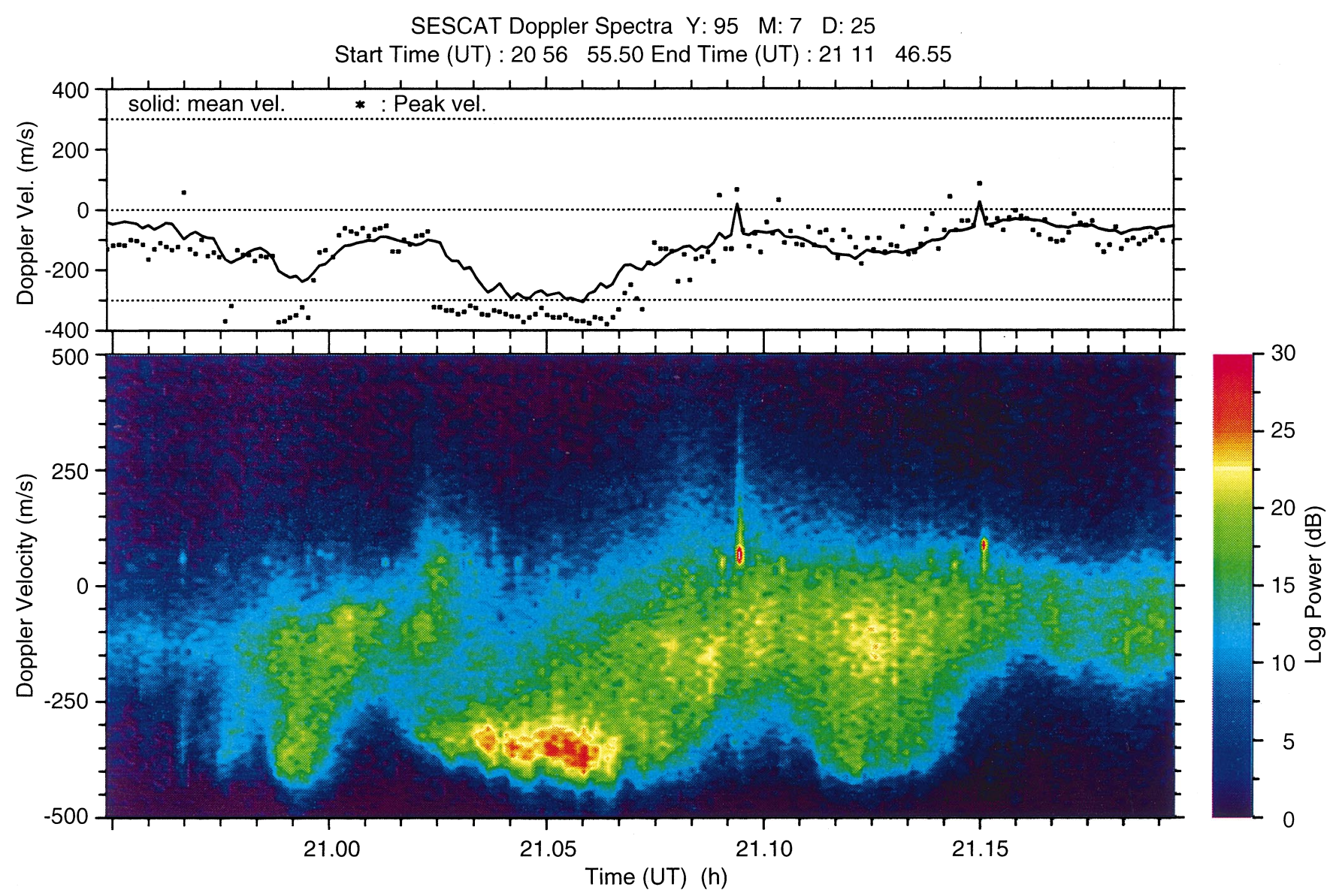

Fig. 4. Another example showing three clear separate cases of type-1 echoes. As seen from the solid line in the top panel, these echoes occur

ionic mass caused by heavy metallic ions such as $\mathrm{Fe}^{+}$ that are known to exist in concentrations perhaps as high as $70-80 \%$ in the nighttime sporadic- $\boldsymbol{E}$-layers (e.g., Behnke and Vikrey, 1975; Steinweg et al., 1992) that are closely associated with the coherent echoes. Figure 8 shows calculated profiles of threshold drift and phase velocities for concentrations of $\mathrm{Fe}^{+}$ions of 0,40 , and $80 \%\left(\bar{m}_{i}=31,41\right.$, and 51 AMU, respectively $)$. Other parameters were taken from the MSIS86 atmospheric model, and the magnetic field is 0.43 Gauss in the SESCAT viewing region. The vertical dashed lines mark the observed range of type-1 velocities. The optimum altitudes for instability are in a small range near $105 \mathrm{~km}$. We see that quite plausible variations in the percentage of long-lived heavy metallic ions at the right altitudes can account for the observed variations in the type-1 wave velocities.

Destabilizing electron-density gradients perpendicular to $\boldsymbol{B}$ and parallel to $\boldsymbol{E}$ could also lower the threshold and hence the observed phase velocity, as suggested first by Farley and Fejer (1975) for the equatorial electrojet. Many authors have pursued this idea for the auroral zone, and Riggin et al. (1986) argued that it could be important at mid-latitudes because of the sharp gradients measured at times in $\boldsymbol{E}_{s}$-layers. during times of ambient electric-field enhancements, presumably to values exceeding the two-stream instability threshold

Our opinion is that metallic ions are more important than gradients, but in any case, it seems that we can satisfactorily explain mean type-1 phase velocities smaller than those observed at the equator. What is not so easy to understand is the existence of electric fields greater than $10 \mathrm{mV} / \mathrm{m}(>15 \mathrm{mV} / \mathrm{m}$ for a phase velocity of $325 \mathrm{~m} / \mathrm{s}$ ), a value far larger than predicted by any mid-latitude dynamo model.

\section{A mechanism for large mid-latitude electric fields}

The similarity of mid-latitude and equatorial type-1 spectral signatures suggests that a similar polarization process might be important in both regions, even though the geometry is quite different. How this process might work was outlined recently by the present authors in a research letter (Haldoupis et al., 1996). For the sake of completeness, we will describe this mechanism again here, but in more detail.

Consider a horizontal, highly conducting $\boldsymbol{E}_{s}$-layer patch at about $105 \mathrm{~km}$ embedded in a low-electrondensity, weakly conducting nighttime $\boldsymbol{E}$ region. We assume that the $\boldsymbol{E}_{s}$ patch has a finite extent in the eastwest (zonal) direction with abrupt boundaries. The east- 
SESCAT Doppler Spectra Y: $93 \quad$ M: 7 D : 12

Start Time (UT) : $1948 \quad 17.18$ End Time (UT) : 195539.58

Norm/zed Power Spectra Averaging Time (s) : 14.75

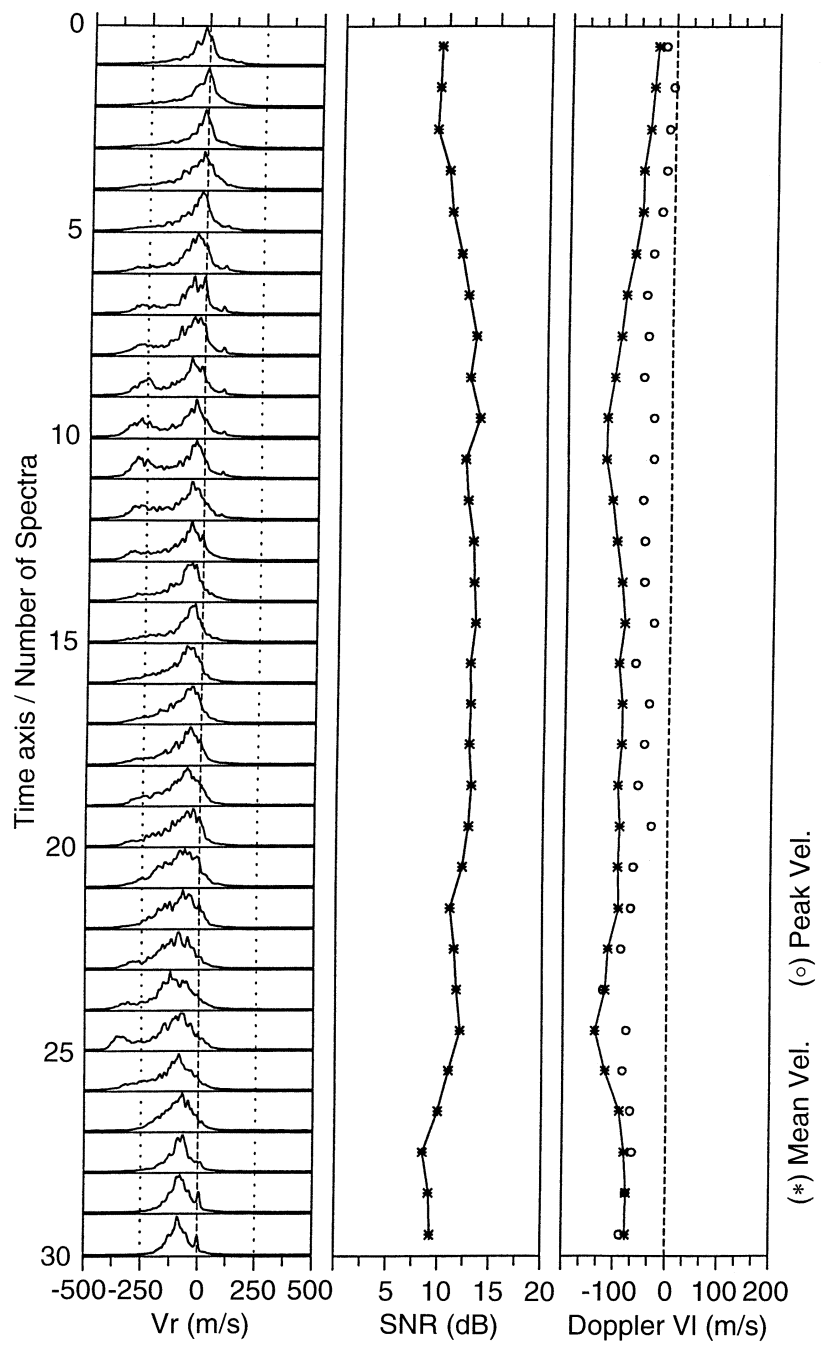

Fig. 5 A time-sequence of self-normalized power spectra showing the appearance for short times of weak type-1 echoes (e.g., spectra 10 and 25 ). These occur during periods of strong type- 2 echo detection and at times when the electric field intensifies to values reaching the twostream instability threshold. This behavior is seen regularly during periods of strong mid-latitude scatter

west direction is perpendicular to the earth's magnetic field $\boldsymbol{B}$, and so this geometry is similar to the situation at the magnetic equator, where the conductivity is sharply bounded above and below, although not by density gradients. Simply put, the equatorial geometry is here "turned on its side". The SESCAT geometry is shown in the sketch at the top of Fig. 9, with $\hat{x}$ pointing horizontally and to the east, $\hat{y}$ pointing towards the radar (downward and southward) and $\hat{z}$ pointing along $\boldsymbol{B}$.

Fig. 7. The distribution of peak phase velocities and spectral width (width/peak ratios, actually) for mid-latitude type-1 echoes. The mean peak velocity is near $285 \mathrm{~m} / \mathrm{s}$, which is about $20 \%$ slower than nominal ion-acoustic speeds at $105 \mathrm{~km}$ and the type- 1 velocities observed at the equator

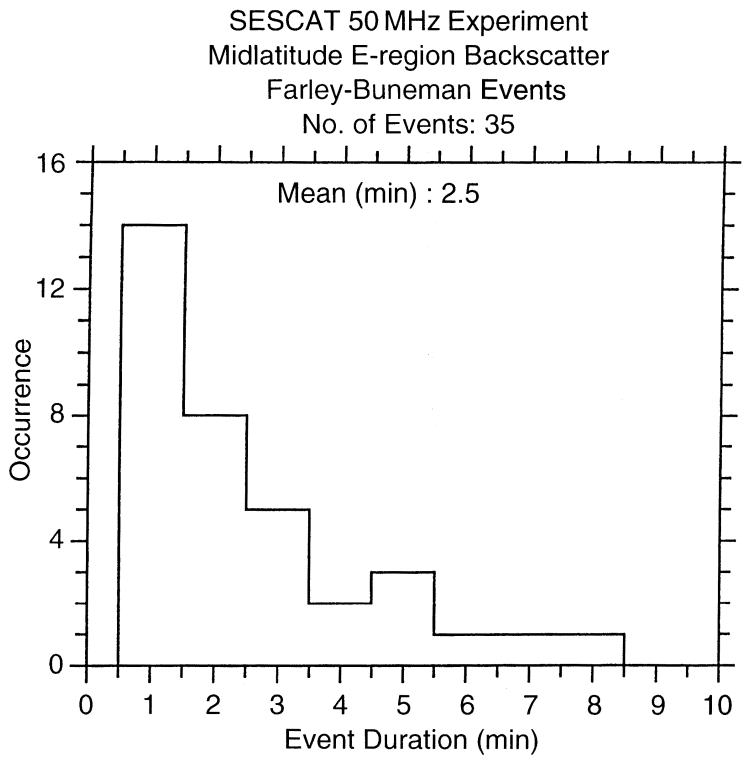

Fig. 6. The distribution of mid-latitude type-1 echo duration based on many events detected by SESCAT. The mean echo lifetime is about $2.5 \mathrm{~min}$
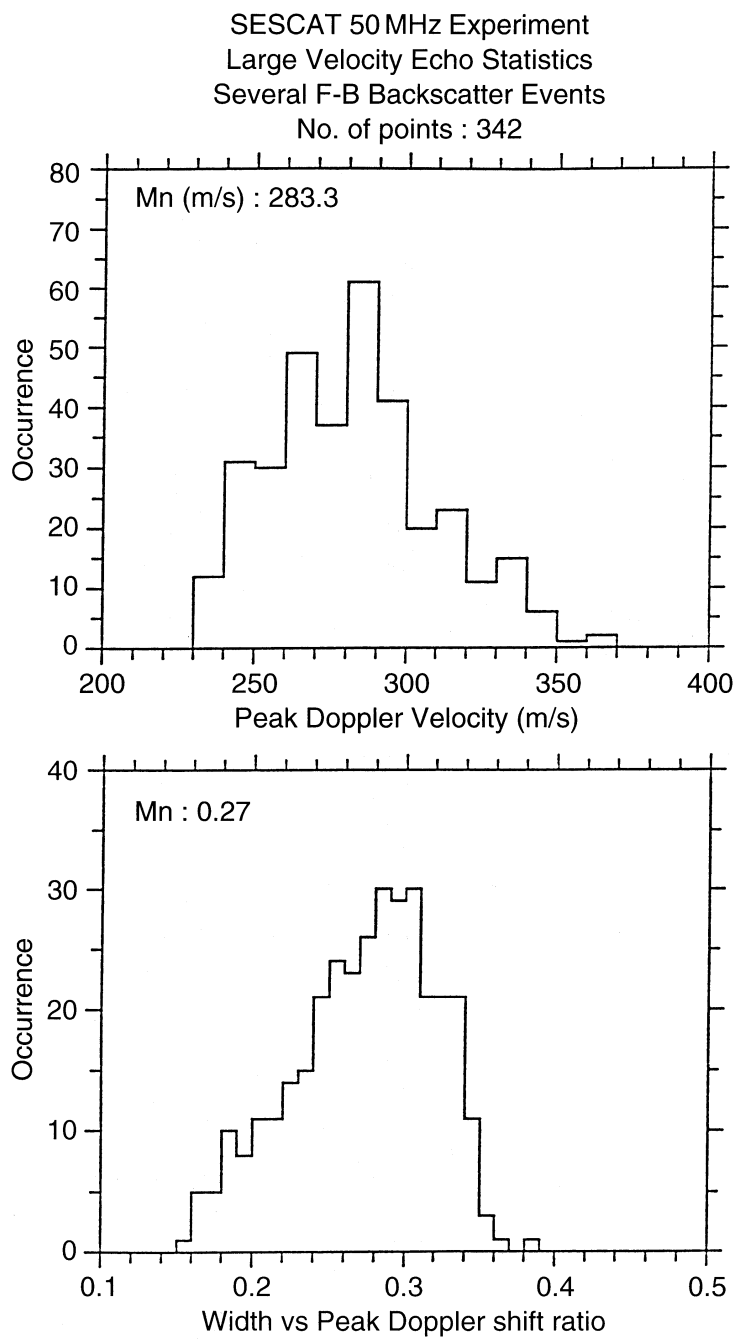

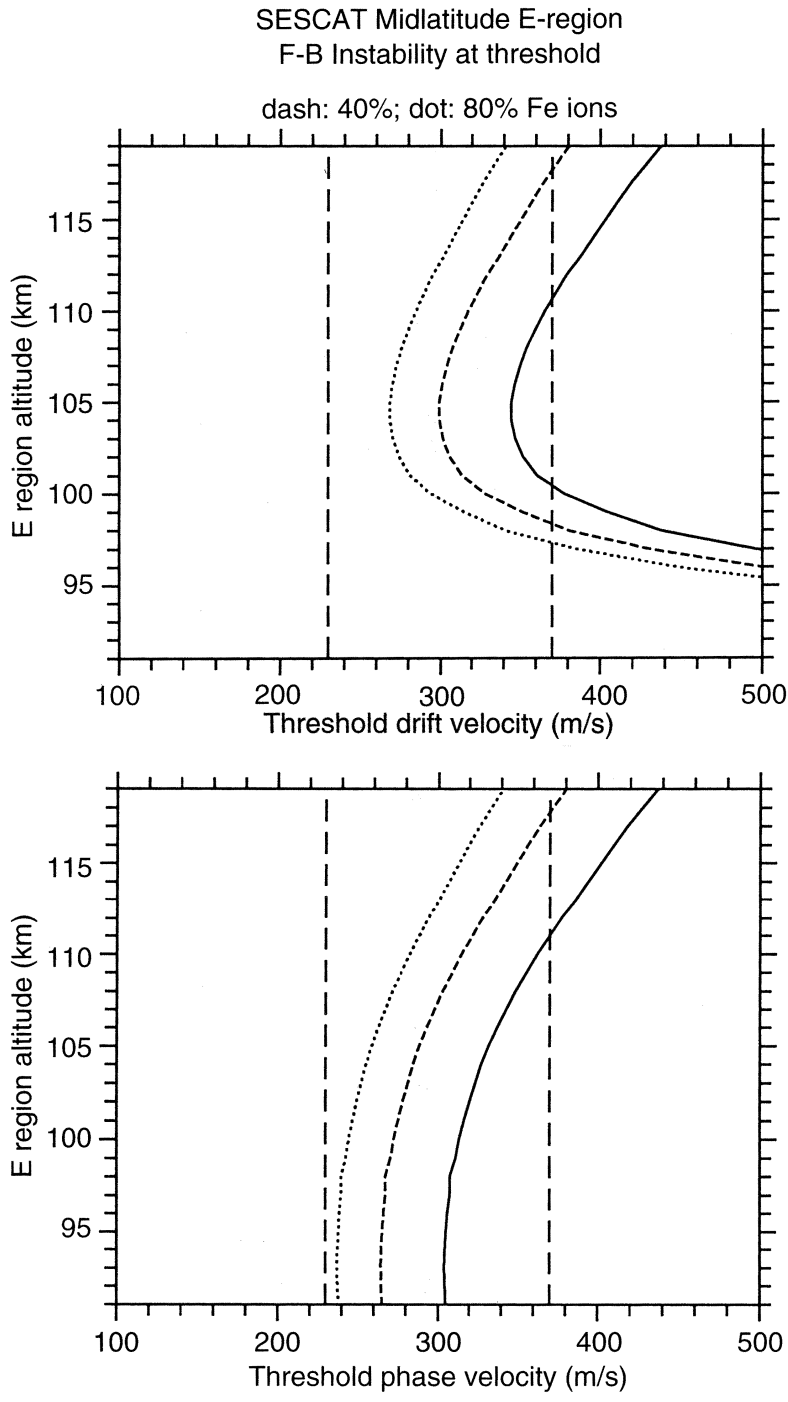

Fig. 8. Linear fluid-theory predictions of the electron-to-ion drift velocity at the two-stream instability threshold (upper panel) and the corresponding phase velocity of type-1 waves. The solid curves are for molecular ions; the dashed and dotted curves are for higher mean ionic masses due to the presence of heavy $\mathrm{Fe}^{+}$ions inside sporadic- $E$-layers. See text for details

In the presence of an ambient ionospheric meridional electric field, a much stronger zonal field will build up inside the patch to maintain divergence-free current flow, just as a vertical polarization field builds up in the equatorial electrojet so that the vertical Hall and Pedersen currents nearly cancel. By the same reasoning, if we assume that zonal currents in the $\boldsymbol{E}_{s}$ patches are almost completely inhibited by the nearly insulating (low-electron-density) zonal-patch boundaries, then a meridional southward/downward electric field $\left(E_{y}^{o}\right)$ will generate a polarization $E_{x}^{p}$ that is larger by nearly the Hall-to-Pedersen-conductivity ratio $\left(\sigma_{H} / \sigma_{p}\right)$. This ratio is about 10 at $105 \mathrm{~km}$ for molecular ions, but can be 20 $40 \%$ higher when metallic ions are important. So the metallic ions are of crucial importance to our proposed explanation: (1) they lower the instability threshold, (2) their dynamics provide the essential patchy geometry,
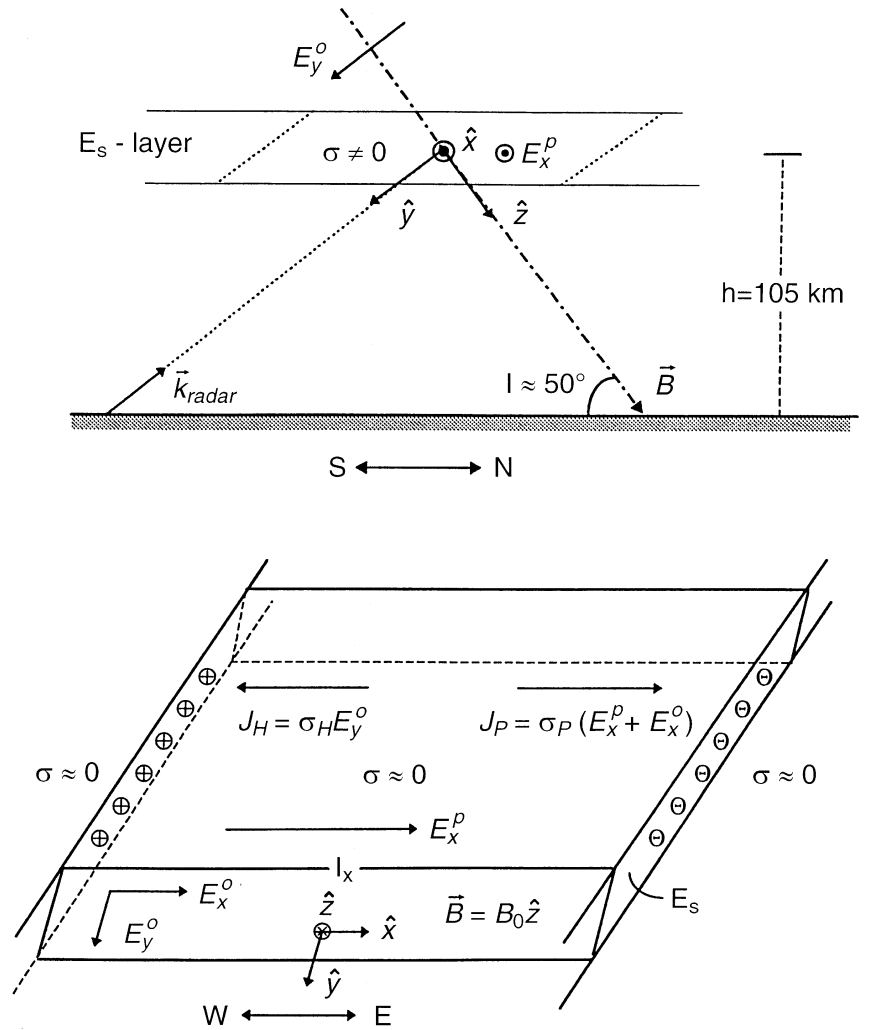

Fig. 9. A sketch of the proposed mid-latitude $E_{s}$ polarization process for the generation of large zonal electric fields, in the meridional (top) and the zonal (bottom) plane. As seen, a meridional electric field, $E_{y}^{o}$, in a plasma slab, leads to the generation of a stronger zonal polarization field as a result of the specific slab geometry and the anisotropy in conductivity in the plane perpendicular to the earth's magnetic field

and (3) they increase the Hall-to-Pedersen-conductivity ratio.

For example, suppose we assume $E_{y}^{o} \simeq 1.2 \mathrm{mV} / \mathrm{m}$, in line with quite summertime fields measured by the Arecibo incoherent-scatter radar (e.g., Kelley, 1989), which observes at about the same $\boldsymbol{L}$-shell as SESCAT. This would produce an eastward $E_{x}^{p}$ of $15-16 \mathrm{mV} / \mathrm{m}$ and a northward and upward electron drift of about $360 \mathrm{~m} / \mathrm{s}$, more than enough to excite F-B waves. This polarity is in line with the great majority of SESCAT type-1 echoes, which have negative Doppler shifts and occur in the premidnight nighttime sector. If $E_{y}^{o}$ reverses direction, so will the polarization field and the sign of the type-1 Doppler shift. As mentioned earlier, such events are very rare, but one example was detected in the early morning hours when the meridional field $\boldsymbol{E}_{y}{ }^{0}$ is known to be directed predominantly poleward (e.g., Kelley, 1989)

There is one important caveat to the arguments just presented: we have ignored possible coupling to the $\boldsymbol{F}$ region via field-aligned currents. If the integrated Pedersen conductivity in the $\boldsymbol{F}$ region above the $\boldsymbol{E}_{s}$ patch is comparable to that of the patch, the effective value of the Hall-to-Pedersen-conductivity ratio, and hence the induced polarization electric field, will be reduced accordingly. This $\boldsymbol{F}$-region coupling should not 
be important for a high-density nightime metallic ion layer (adding a fourth point to the three listed above), but might be for a weak layer.

\section{Discussion}

Our model seems to be able to account quite nicely for the SESCAT observations of type-1 echoes. There are some remaining questions, however: (1) Why do we not see type-1 echoes more often? (2) How do the more or less vertical electron drift currents ( the Hall currents driven by the polarization field) close? The answer to (1) is most likely that we encounter the required geometry only rarely. The metallic-ion sporadic- $\boldsymbol{E}$ layer has to be centered near $105 \mathrm{~km}$ and has to have quite sharp zonal boundaries so that the zonal Hall current is strongly inhibited. Furthermore, there must be no easy way for this current to close via some magnetic field-aligned path. On the other hand, the vertical electron drift currents presumably do close via field-aligned currents to other regions, perhaps even in the opposite hemisphere. Even though these vertical current densities might be quite high locally, this would be true only in isolated small patches of the ionosphere and so the current would not be noticed in magnetic data. In any case, the closure details are not important to our discussion here; all that matters is that the vertical currents $\boldsymbol{d o}$ close, and so inhibiting polarizations charges do not build up on the top and bottom of the $\boldsymbol{E}_{s}$-layers.

Finally, we ask whether the key assumption in this model, the existence of dense sporadic- $\boldsymbol{E}$-layers having a finite zonal extent with sharp boundaries, is plausible? Various past studies with ionosondes, rockets, and other techniques show a great deal of $\boldsymbol{E}_{s}$ patchiness in the horizontal plane with characteristic gradient lengths of a few kilometers or less (e.g., see review by Whitehead, 1989). Direct estimates of horizontal $\boldsymbol{E}_{s}$ structuring, obtained from incoherent-scatter measurements by Miller and Smith (1978) with the Arecibo radar, show localized ionization clouds with sharp azimuthal boundaries and gradients. In a recent paper by Kelley $\boldsymbol{e t}$ al. (1995), a pronounced wavelike horizontal structure, with sharp enhancements and depletions in plasma density, was detected with a low-altitude rocket launched into a mid-latitude sporadic- $\boldsymbol{E}$-layer with a peak electron density near $10^{6} \mathrm{~cm}^{-3}$. Finally, very recent interferometer studies with the Chung-Li radar in Taiwan by Huang et al. (1995) show that mid-latitude $\boldsymbol{E}$-region echoes originate in localized patches with cross-field widths of about $2-4 \mathrm{~km}$.

In summary, the model we have presented seems to have no obvious and major flaws and accounts qualitatively and at least semi-quantitatively for the SESCAT radar observations. It would be nice to have a more quantitative model of the currents and fields inside a sporadic- $\boldsymbol{E}$ patch and its surroundings, but constructing a realistic model may be difficult. The ideal test would be a rocket measurement of the electric field inside the layer when type-1 radar echoes were being observed. The odds against getting such a rocket in the right place at the right time, however, seem formidable to say the least.

Acknowledgements. SESCAT is operated in Crete by the MaxPlanck-Institut für Aeronomie, Germany and the University of Crete, Greece. Partial support for the completion of this work was provided by PENED-96 program of GGET, Greek Ministry of Research and Technology. We wish to thank D. Zouridis and T. Grispolakis for their assistance in SESCAT operation and data collection. The cooperation of the Technical College of Iraklion (TEI) and the Polytechnic University of Chanea in allowing the use of their land for the SESCAT sites is also greatfully acknowledged. One of us (DF) acknowledges with thanks the support and hospitality of the Alexander von Humboldt Foundation and the Max-Planck-Institut für Aeronomie during the period in which this work was done.

Topical Editor D. Alcayde thanks A. Bourdillon for his help in evaluating this paper.

\section{References}

Behnke, R. A., and J. F. Vikrey, Radar evidence for $\mathrm{Fe}^{+}$in a sporadic E-layer, Radio Sci., 10, 325, 1975.

Buneman, O., Excitation of field-aligned sound waves by electron streams, Phys. Rev. Lett., 10, 285, 1963.

Ecklund, W. L., D. A. Carter, and B. B. Balsley, Gradient drift irregularities in mid-latitude sporadic E, J. Geophys, Res.,86, $858,1981$.

Farley, D. T., A plasma instability resulting in field-aligned irregularities in the ionosphere, J. Geophys. Res., 68, 6083, 1963.

Farley, D. T., Theory of equatorial electrojet plasma waves: new developments and current status, J. Atmos. Terr. Phys., 47, 729, 1985.

Farley, D. T., and B. G. Fejer, The effect of the gradient drift term on the type-1 electrojet irregularities, J. Geophys. Res., 80, 3087, 1975.

Fejer, B. G., and M. C. Kelley, Ionospheric irregularities, Rev. Geophys., 18, 401, 1980.

Haldoupis, C., A review of radio studies of auroral $\boldsymbol{E}$-region ionosphere, Ann. Geophysicae, 7, 239, 1989.

Haldoupis, C., and K. Schlegel, A 50-MHz radio Doppler experiment for mid-latitude $\boldsymbol{E}$-region coherent backscatter studies: System description and first results, Radio Sci. 28, 959, 1993.

Haldoupis, C., and K. Schlegel, Characteristics of mid-latitude coherent backscatter from the ionospheric $\boldsymbol{E}$-region obtained with SESCAT, J. Geophys. Res., 101, 3387, 1996.

Haldoupis, C., K. Schlegel, and D. T. Farley, An explanation for type-1 echoes from the mid-latitude $\boldsymbol{E}$-region ionosphere, Geophys. Res. Lett., 23, 97, 1996.

Huang, C.-M, E. Kudeki, S. J. Franke, C. H. Liu, and J. Röttger, Brightness distribution of mid-latitude $\boldsymbol{E}$-region echoes detected at the Chung-Li VHF radar, J. Geophys. Res., 100, 14703, 1995.

Kelley, M. C., The Earth's Ionosphere: Plasma Physics and Electrodynamics, Academic, San Diego, Calif., 1989.

Kelley, M. C., D. Riggin, R. F. Pfaff, W. E. Swartz, J. F. Providakes, and C.-S. Huang, Large amplitude quasi-periodic fluctuations associated with a mid-latitude sporadic $\boldsymbol{E}$-layer, $\boldsymbol{J}$. Atmos. Terr. Phys., 57, 1165, 1995.

Keohler, J. A., G. J. Sofko, and V. Mahta, A statistical study of magnetic aspect angle effects associated with VHF auroral backscatter, Radio Sci., 20, 689, 1985.

Miller, K. L., and L. G. Smith, Incoherent scatter radar observations of irregular structure in mid-latitude sporadic $\boldsymbol{E}$ layers, $\boldsymbol{J}$. Geophys. Res., 83, 3761, 1978.

Riggin, D., W. E. Swartz, J. F. Providakes, and D. T. Farley, Radar studies of long-wavelength waves associated with mid-latitude sporadic $\boldsymbol{E}$ layers, J. Geophys. Res., 91, 8011, 1986.

Roble, R. G., and E. C. Ridley, A thermosphere-ionosopheremesosphere electrodynamics general circulation model (time- 
GCM): Equinox solar cycle minimum simulations $(30-500 \mathrm{~km})$, Geophys. Res. Lett., 21, 417, 1994.

Schlegel, K., The influence of metallic ions on the plasma instabilities in the high-latitude $\boldsymbol{E}$ region, Radio $\boldsymbol{S c i . ,} \mathbf{2 0}, \mathbf{7 4 0 ,}$ 1985.

Schlegel, K., and C. Haldoupis, Observation of the modified twostream plasma instability in the mid-latitude $\boldsymbol{E}$-region ionosphere, J. Geophys. Res., 99, 6219, 1994.

Steinweg, A., D. Krankowsky, P. Lämmerzahl, and B. Anweilwer, Metal ion layers in the auroral lower $\boldsymbol{E}$ region measured by mass spectrometers, J. Atmos. Terr. Phys., 54, 703, 1992.

Sudan, R. N., Unified theory of type-I and type-II irregularities in the equatorial electrojet, J. Geophys. Res., 88, 4853, 1983.
Tsunoda, R. T., S. Fukao, and M. Yamamoto, On the origin of quasi-periodic radar backscatter from mid-latitude sporadic $\boldsymbol{E}$, Radio Sci., 29, 349, 1994.

Whitehead, J. D., Recent work on mid-latitude and equatorial sporadic- E, J. Atmos. Terr.Phys., 51, 401, 1989.

Woodmann, R. F., M. Yamamoto, and S. Fukao, Gravity wave modulation of gradient drift instabilities in mid-latitude sporadic-E irregularities, Geophys. Res. Lett., 18, 1197, 1991.

Yamamoto, M., S. Fukao, R. F. Woodman, T. Ogawa, T. Tsuda, and $\mathbf{S}$. Kato, Mid-latitude $\boldsymbol{E}$-region field-aligned irregularities observed with the MU radar, J. Geophys. Res., 96, 15963, 1991. 